\title{
Probing Resonance Decays to Two Visible and Multiple Invisible Particles
}

\author{
Won Sang Cho, ${ }^{1}$ Doojin Kim, ${ }^{2}$ Konstantin T. Matchev, ${ }^{1}$ and Myeonghun Park ${ }^{3}$ \\ ${ }^{1}$ Physics Department, University of Florida, Gainesville, Florida 32611, USA \\ ${ }^{2}$ Department of Physics, University of Maryland, College Park, Maryland 20742, USA \\ ${ }^{3}$ CERN, Theory Division, CH-1211 Geneva 23, Switzerland
}

(Received 14 June 2012; revised manuscript received 6 March 2014; published 27 May 2014)

\begin{abstract}
We consider the decay of a generic resonance to two visible particles and any number of invisible particles. We show that the shape of the invariant mass distribution of the two visible particles is sensitive to both the mass spectrum of the new particles, as well as the decay topology. We provide the analytical formulas describing the invariant mass shapes for the nine simplest topologies (with up to two invisible particles in the final state). Any such distribution can be simply categorized by its end point, peak location, and curvature, which are typically sufficient to discriminate among the competing topologies. In each case, we list the effective mass parameters which can be measured by experiment. In certain cases, the invariant mass shape is sufficient to completely determine the new particle mass spectrum, including the overall mass scale.
\end{abstract}

DOI: 10.1103/PhysRevLett.112.211801

The dark matter problem and the mystery of the feeble neutrinos greatly motivate the ongoing LHC searches for new physics in channels with missing energy. Alas, at hadron colliders like the LHC, deciphering events with invisible particles in the final state is notoriously difficult.

The problem is schematically illustrated in Fig. 1, which depicts the generic decay of some new heavy resonance $A$ into $N_{v}$ visible particles $v_{i}$ and $N_{\chi}$ "invisible" particles $\chi_{i}$ (neutrinos or dark matter candidates) which leave no trace in the detector. A priori, we have no way of knowing the underlying physics behind Fig. 1, and thus, we are missing the answers to some very basic questions: (1) How many invisible particles are in the final state? (2) What are their masses? (3) What is the exact topology (i.e., Feynman diagram) of Fig. 1: are there any intermediate resonances, and if so, what are their masses?

Historically, the topic of mass measurements has attracted the most attention in the literature (for a review, see [1]). Unfortunately, virtually all proposed methods suffer from two drawbacks. First, one must typically assume the correct decay topology for Fig. 1, including the correct number $N_{\chi}$ of invisible particles. If this guess is incorrect, the method does not apply. This motivates us to address the issue of the correct decay topology and number of invisible particles concurrently with (perhaps even prior to) the more traditional question of mass measurements [2]. Second, most methods for mass measurements utilize kinematic end points, where the available statistics can be rather poor (in the sense that the most populated bins are rarely near the kinematic end point). Here, we shall, instead, concentrate on the region near the peak rather than the end point of the kinematic distribution. (The exact shape in the vicinity of the end point does contain information about $N_{\chi}[3,4]$, but is difficult to measure
PACS numbers: 13.85.Rm, 14.60.Lm, 14.80.Ly, 95.35.+d

precisely in the presence of backgrounds and detector effects.) Our main result will be the derivation of the analytical formulas necessary to analyze the full shape of the invariant mass distributions of the visible particles in Fig. 1, including the location of the peak. We shall then demonstrate how those results can be used to determine (1) the number of missing particles, (2) their masses, and (3) the associated event topology.

Our setup is as follows. We consider the generic decay from Fig. 1 without any prior assumptions about the decay topology or the number of invisible particles. As seen in Table I, the number of inequivalent decay topologies proliferates very quickly as we increase the number of particles in the final state. Let us begin with the simplest and most challenging case of $N_{v}=2$, postponing $N_{v}>2$ to a future study [5]. According to Table I, there are two topologies with $N_{\chi}=1$, shown in Figs. 2(a) and 2(b), and seven topologies with $N_{\chi}=2$, shown in Figs. 2(c)-2(i). Our main goal is to analyze and contrast the $v_{1} v_{2}$ invariant mass distribution [6] in each of those nine cases.

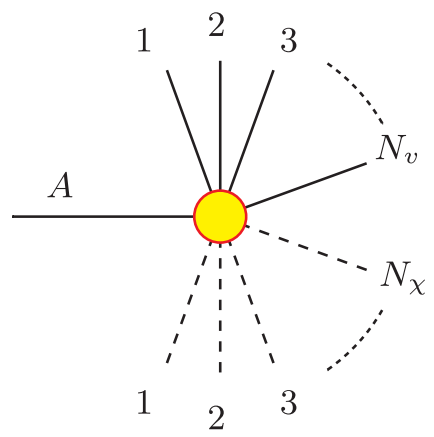

FIG. 1 (color online). The generic decay topology under consideration. 
TABLE I. The number of inequivalent event topologies as a function of $1 \leq N_{v} \leq 4$ and $1 \leq N_{\chi} \leq 5$.

\begin{tabular}{lccccc}
\hline \hline \multicolumn{6}{c}{$N_{\chi}$} \\
\hline$N_{v}$ & 1 & 2 & 3 & 4 & 5 \\
\hline 1 & 1 & 2 & 4 & 8 & 16 \\
2 & 2 & 7 & 20 & 55 & 142 \\
3 & 4 & 20 & 78 & 270 & 860 \\
4 & 8 & 55 & 270 & 1138 & 4294 \\
\hline \hline
\end{tabular}

The differential distribution of the invariant mass $m \equiv m_{v_{1} v_{2}}$ will be described by an analytical formula

$$
\frac{d N}{d m} \equiv f\left(m ; M_{A}, M_{B_{i}}, M_{\chi_{j}}\right),
$$

which is only a function of the unknown masses [8]. Given the general formula (1) for $f(m)$, we can easily obtain its kinematic end point

$$
E \equiv \max \{m\}
$$

and the location $P$ of the peak of the $f(m)$ distribution

$$
f(m=P) \equiv \max \{f(m)\} .
$$

Let us also define the dimensionless derivative ratios

$$
R_{n} \equiv-\left(\frac{m^{n}}{f(m)} \frac{d^{n} f(m)}{d m^{n}}\right)_{m=P} .
$$

By definition, $R_{1}=0$, as long as $f(m)$ is continuously differentiable at $m=P$, while $R_{2}$ parametrizes the curvature of $f(m)$ at $m=P$.

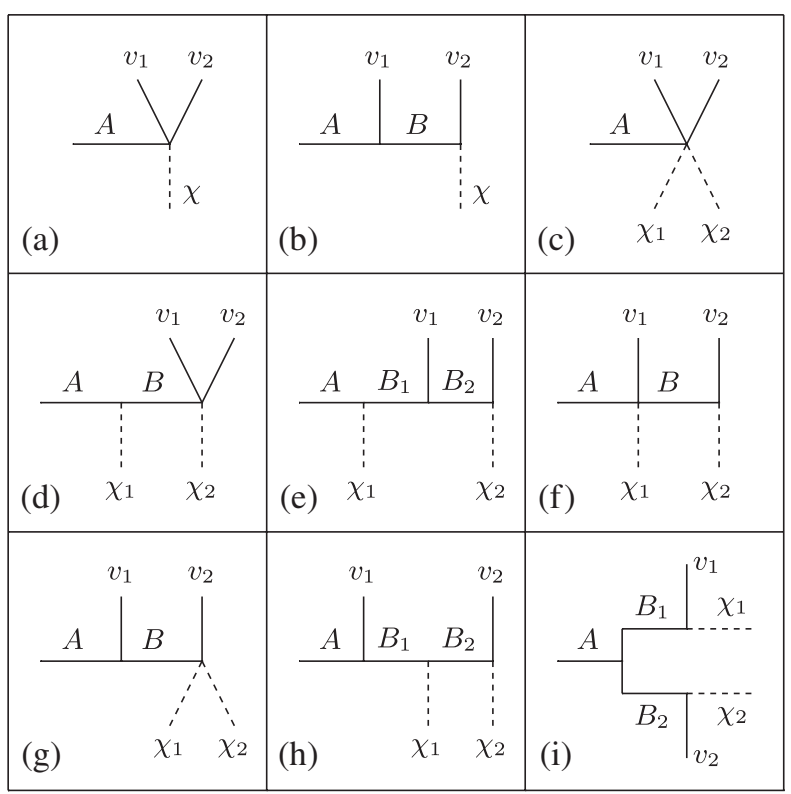

FIG. 2. The nine $N_{v}=2$ topologies with $N_{\chi} \leq 2$.
The parameters $E, P$, and $R_{n}$ are in principle all experimentally measurable from the distribution (1). Traditional studies [11] have always concentrated on measuring just the end point $E$, failing to utilize all of the available information encoded in the distribution $f(m)$. The end point approach gives a single measurement (2), which is clearly insufficient to determine the full spectrum of resonances involved in the decay chain of Fig. 1. Here, we propose to invoke the full shape (1) in the analysis [9]. We envision that, in practice, this will be done by performing unbinned maximum-likelihood fits of (1) to the observed data. In order to illustrate the power of the method here, it is sufficient to consider just the additional individual measurements of $P$ and $R_{2}$. Since they are obtained from the most populated bins near the peak, we can expect that they will be rather well measured. More importantly, the additional information about $P$ and $R_{2}$ might be sufficient to completely determine the mass spectrum [see Eqs. (13) and (14) below]. But first, we need to present our results for (1)-(4) in each of the nine cases in Fig. 2.

The topology of Fig. 2(a).-For a three body decay to massless visible particles, one has

$$
f\left(m ; M_{A}, M_{\chi}\right) \sim m \lambda^{1 / 2}\left(m^{2}, M_{A}^{2}, M_{\chi}^{2}\right),
$$

where

$$
\lambda(x, y, z) \equiv x^{2}+y^{2}+z^{2}-2 x y-2 y z-2 x z .
$$

In this case,

$$
\begin{gathered}
E=M_{A}-M_{\chi}, \\
P=\left[2 M_{A} M_{\chi}\left(2-\sqrt{1+3 \alpha^{2}}\right) /(3 \alpha)\right]^{1 / 2}, \\
R_{2}=6\left[1+\left(1+3 \alpha^{2}\right)^{-1 / 2}\right]^{-1},
\end{gathered}
$$

where

$$
\alpha \equiv 2 M_{A} M_{\chi} /\left(M_{A}^{2}+M_{\chi}^{2}\right) .
$$

Contrary to popular belief, one can now solve for both masses $M_{A}$ and $M_{\chi}$, given two of the three measurements (7)-(9). For example, using the peak location $P$ and the end point $E$, we find

$$
\begin{aligned}
& M_{A}=\frac{E}{2}\left(\frac{P}{E} \sqrt{\frac{2-3(P / E)^{2}}{1-2(P / E)^{2}}}+1\right), \\
& M_{\chi}=\frac{E}{2}\left(\frac{P}{E} \sqrt{\frac{2-3(P / E)^{2}}{1-2(P / E)^{2}}}-1\right) .
\end{aligned}
$$

Equations (11) and (12) offer a new method of determining both $M_{A}$ and $M_{\chi}$, which is a simpler alternative to the $M_{T 2}$ 
kink method of [12], since, here, we do not rely on the $E_{T}$ measurement at all, and do not require to reconstruct the decay chain on the other side of the event.

In fact, one does not even need an end point measurement, since the peak location $P$ and the curvature $R_{2}$ are sufficient for this purpose

$$
\begin{aligned}
& M_{A}=\frac{P}{\sqrt{2}}\left(\frac{6-R_{2}}{4-R_{2}}+\sqrt{\frac{12-R_{2}}{4-R_{2}}}\right)^{1 / 2}, \\
& M_{\chi}=\frac{P}{\sqrt{2}}\left(\frac{6-R_{2}}{4-R_{2}}-\sqrt{\frac{12-R_{2}}{4-R_{2}}}\right)^{1 / 2} .
\end{aligned}
$$

Note that, in analogy to the matrix element method [13], Eqs. (13) and (14) are capable of determining the complete mass spectrum in the decay chain of a resonance decaying semi-invisibly as in Fig. 2(a), without relying on any kinematic end point measurements.

In order to get a rough idea of the precision of these mass determinations, in Fig. 3 on the left (right) we show the results from 10000 pseudoexperiments with 100 (1000) signal events each. In each pseudoexperiment, the two masses $M_{A}$ and $M_{\chi}$ are extracted from a maximum-likelihood fit of the simulated data to the full distribution (5). Figure 3 shows that, as expected, the mass difference is measured quite well, at the level of $\sim 1 \%$ with just 100 events. At the same time, the mass sum (or equivalently, the absolute mass scale) is also being determined, albeit less precisely, at the level of $30 \%(\sim 10 \%)$ with 100 (1000) events.

The topology of Fig. 2(b).- - Here, one obtains the celebrated triangular shape

$$
\begin{gathered}
f(m) \sim m, \\
E=P=\sqrt{\left(M_{A}^{2}-M_{B}^{2}\right)\left(1-M_{\chi}^{2} / M_{B}^{2}\right)}, \\
R_{2}=\infty .
\end{gathered}
$$
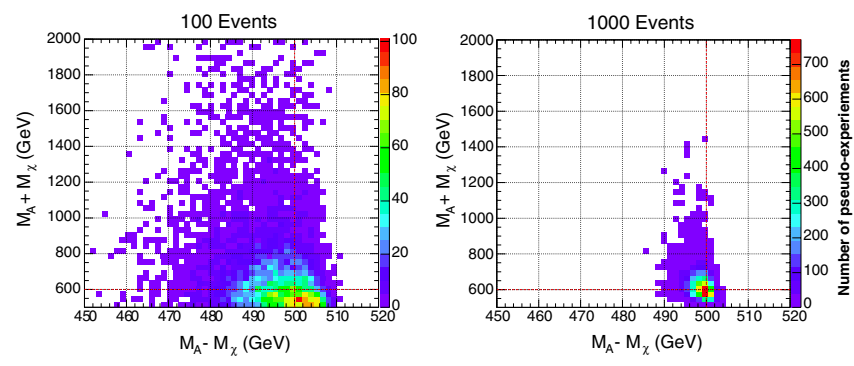

FIG. 3 (color online). Distribution of $M_{A}$ and $M_{\chi}$ found by a maximum-likelihood fit to Eq. (5) in 10000 pseudoexperiments with 100 signal events (left) or 1000 signal events (right). The input study point has $M_{A}=550 \mathrm{GeV}$ and $M_{\chi}=50 \mathrm{GeV}$.
Unfortunately, the masses enter the shape (15) only through the combination (16), which is the single effective mass parameter accessible experimentally.

The topology of Fig. 2(c).-The shape is more conveniently given in integral form, which is easy to code up

$$
\begin{gathered}
f(m) \sim m \int_{\left(M_{\chi_{1}}+M_{\chi_{2}}\right)^{2}}^{\left(M_{A}-m\right)^{2}} \frac{d s}{s} \sqrt{\lambda\left(M_{A}^{2}, m^{2}, s\right) \lambda\left(s, M_{\chi_{1}}^{2}, M_{\chi_{2}}^{2}\right)}, \\
E=M_{A}-M_{\chi_{1}}-M_{\chi_{2}} .
\end{gathered}
$$

The explicit formulas for $P$ and (18) will be shown in [5]. The important point is that, in principle, all three masses $M_{A}, M_{\chi_{1}}$, and $M_{\chi_{2}}$ can be simultaneously determined from a fit of Eq. (18) to the data, just like in Fig. 3 [5].

The topology of Fig. 2(d).-The invariant mass distribution of the visible particles $v_{1}$ and $v_{2}$ is not affected by the emission of invisible particles upstream, and so, this case is equivalent to the topology of Fig. 2(a). The corresponding results can be obtained from (5)-(9) with the substitution $A \rightarrow B$, since now the role of the parent resonance is played by the intermediate particle $B$. One would then be able to determine independently $M_{B}$ and $M_{\chi_{2}}$, while $M_{A}$ and $M_{\chi_{1}}$ would remain unknown.

The topology of Fig. 2(e).- Similarly, this case is equivalent to Fig. 2(b), with the substitutions $A \rightarrow B_{1}$, $B \rightarrow B_{2}$, and $\chi \rightarrow \chi_{2}$. Once again, the emission of the invisible particle $\chi_{1}$ upstream is not observable. The only measurable parameter in this case will be the end point $E$.

The topology of Fig. 2(f).-We find

$$
\begin{aligned}
f(m) \sim & \left.m \int_{M_{B}^{2}\left(1+\frac{m^{2}}{M_{B}^{2}-M_{\chi_{2}}^{2}}\right)}^{\left(M_{A}-M_{\chi_{1}}\right)^{2}} \frac{d s}{s} \sqrt{\lambda\left(s, M_{A}^{2}, M_{\chi_{1}}^{2}\right.}\right), \\
\sim & -m\left[K_{+} K_{-}+\frac{1}{2}\left(X_{+}^{2}+X_{-}^{2}\right) \ln \left(\frac{K_{+}+K_{-}}{K_{+}-K_{-}}\right)\right. \\
& \left.+X_{+} X_{-} \ln \left(\frac{X_{-} K_{+}-X_{+} K_{-}}{X_{-} K_{+}+X_{+} K_{-}}\right)\right],
\end{aligned}
$$

where

$$
\begin{gathered}
X_{ \pm} \equiv M_{A} \pm M_{\chi_{1}}, \quad K_{ \pm} \equiv \sqrt{X_{ \pm}^{2}-K^{2}(m)}, \\
K^{2}(m) \equiv M_{B}^{2}\left(1+\frac{m^{2}}{M_{B}^{2}-M_{\chi_{2}}^{2}}\right), \\
E=\sqrt{\left[\left(M_{A}-M_{\chi_{1}}\right)^{2}-M_{B}^{2}\right]\left(1-M_{\chi_{2}}^{2} / M_{B}^{2}\right)} .
\end{gathered}
$$

In this case, out of the four input masses entering the topology of Fig. 2(f), one can measure 3 independent degrees of freedom, e.g., $M_{A} / M_{B}, M_{\chi_{1}} / M_{B}$, and $M_{B}^{2}-M_{\chi_{2}}^{2}$. 
The topology of Fig. 2(g).--The shape is described by

$$
f(m) \sim m \int_{\left(M_{\chi_{1}}+M_{\chi_{2}}\right)^{2}}^{M_{B}^{2}\left(1-\frac{m^{2}}{M_{A}^{2}-M_{B}^{2}}\right)} \frac{d s}{s} \sqrt{\lambda\left(s, M_{\chi_{1}}^{2}, M_{\chi_{2}}^{2}\right)},
$$

and it is easy to see that the results are obtained from (20)-(23) with the substitution $M_{A} \leftrightarrow-M_{\chi_{2}}$. In particular, the three measurable parameters, in this case, can be taken as $M_{\chi_{1}} / M_{B}, M_{\chi_{2}} / M_{B}$, and $M_{A}^{2}-M_{B}^{2}$.

The topology of Fig. 2(h).-This is the "sandwich" topology studied in [14]. The shape is given by

$$
\begin{gathered}
f(m) \sim \begin{cases}\eta m, & 0 \leq m \leq e^{-\eta} E, \\
m \ln (E / m), & e^{-\eta} E \leq m \leq E,\end{cases} \\
\eta \equiv \cosh ^{-1}\left(\frac{M_{B_{1}}^{2}+M_{B_{2}}^{2}-M_{\chi_{1}}^{2}}{2 M_{B_{1}} M_{B_{2}}}\right),
\end{gathered}
$$

and

$$
\begin{gathered}
E=\left[e^{\eta}\left(M_{A}^{2}-M_{B_{1}}^{2}\right)\left(M_{B_{2}}^{2}-M_{\chi_{2}}^{2}\right) /\left(M_{B_{1}} M_{B_{2}}\right)\right]^{1 / 2}, \\
P=\left\{\begin{array}{ll}
E e^{-\eta}, & \eta<1 ; \\
E e^{-1}, & \eta \geq 1 ;
\end{array} \quad R_{2}= \begin{cases}\infty, & \eta<1 ; \\
1, & \eta \geq 1 .\end{cases} \right.
\end{gathered}
$$

The distribution (25) exhibits a cusp at the nondifferentiable point $m=e^{-\eta} E$. In this case, there are five mass inputs: $M_{A}, M_{B_{1}}, M_{B_{2}}, M_{\chi_{1}}$, and $M_{\chi_{2}}$, but only two independent measurable parameters: $\eta$ and $E$.

The topology of Fig. 2(i).-This is the "antler" topology which was studied in [15] for the symmetric case of $M_{B_{1}}=$ $M_{B_{2}}$ and $M_{\chi_{1}}=M_{\chi_{2}}$. Here, we generalize the result in [15] to arbitrary masses and find that $f(m)$ is given by the same expression (25), only this time

$$
\begin{gathered}
\eta \equiv \cosh ^{-1}\left(\frac{M_{A}^{2}-M_{B_{1}}^{2}-M_{B_{2}}^{2}}{2 M_{B_{1}} M_{B_{2}}}\right), \\
E=\left[e^{\eta}\left(M_{B_{1}}^{2}-M_{\chi_{1}}^{2}\right)\left(M_{B_{2}}^{2}-M_{\chi_{2}}^{2}\right) /\left(M_{B_{1}} M_{B_{2}}\right)\right]^{1 / 2},
\end{gathered}
$$

and identical expressions (28) for $P$ and $R_{2}$. Just like the case of Fig. 2(h), out of the five mass inputs, $\eta$ and $E$ are the only two measurable mass parameters. Table II summarizes the final tally of input particle masses and independent measurable parameters for each topology.

TABLE II. The number of mass inputs $N_{m}$ for each topology in Fig. 2 and the number of independent measurable parameters $N_{p}$ in the definition of $f(m)$.

\begin{tabular}{lccccc}
\hline \hline Topology & (a),(d) & (b),(e) & (c) & (f),(g) & (h),(i) \\
\hline$N_{m}$ & 2 & 3 & 3 & 4 & 5 \\
$N_{p}$ & 2 & 1 & 3 & 3 & 2 \\
\hline \hline
\end{tabular}

Each topology from Fig. 2 also maps onto a restricted region in the $\left(R_{2}, P / E\right)$ plane, as shown in Fig. 4 [for convenience, instead of $R_{2} \in(0, \infty)$, in the figure, we plot $\left.(2 / \pi) \tan ^{-1} R_{2} \in(0,1)\right]$. For example, the cyan circle at $(1,1)$ marks the prediction for the two topologies of Figs. 2(b) and 2(e), while the magenta dot at $(0.5,0.37)$ and the magenta vertical line correspond to the two topologies of Figs. 2(h) and 2(i). The blue (red, green, black) points refer to the topologies of Figs. 2(a) and 2(d) [Figs. 2(g), 2(f) and 2(c)]. Figure 4 demonstrates that with the three measurements $E, P$, and $R_{2}$, one can already begin to constrain qualitatively the allowed event topologies.

In fact, one can do even better by fitting to the full invariant mass shapes derived here. For illustration, we consider a scenario where particle $A$ is a vector boson $(V)$, $B$ is a fermion $(F)$, and $C$ is another vector boson $(V)$, and study two representative event topologies. The blue squares in Fig. 5 correspond to the antler topology case of Fig. 2(i) for which the $m$ distribution exhibits a cusp at $m=e^{-\eta} E$ [see Eq. (25)]. This example was considered in [15] for the purpose of measuring the masses, which were chosen as $M_{A}=1500 \mathrm{GeV}, \quad M_{B_{1}}=M_{B_{2}}=730 \mathrm{GeV}$ and $M_{\chi_{1}}=M_{\chi_{2}}=100 \mathrm{GeV}$. We also consider one cuspless case, namely, the topology of Fig. 2(a) with a mass spectrum $M_{A}=550 \mathrm{GeV}, M_{\chi}=400 \mathrm{GeV}$ (red circles in Fig. 5).

Figure 5 shows the average $p$ values $(\bar{P})$ obtained in 200 pseudoexperiments, with 500 events each. For each example, the filled symbols represent the case in which spin correlations are absent; i.e., the "data" are sampled from the phase space (PS) distributions derived earlier. We see that the fit clearly prefers the correct topologies from Figs. 2(i) and 2(a) [and their identical twins from Figs. 2(h) and 2(d)], while the wrong topologies are disfavored.

In models in which the fermions have chiral couplings, the invariant mass shapes considered here will be slightly

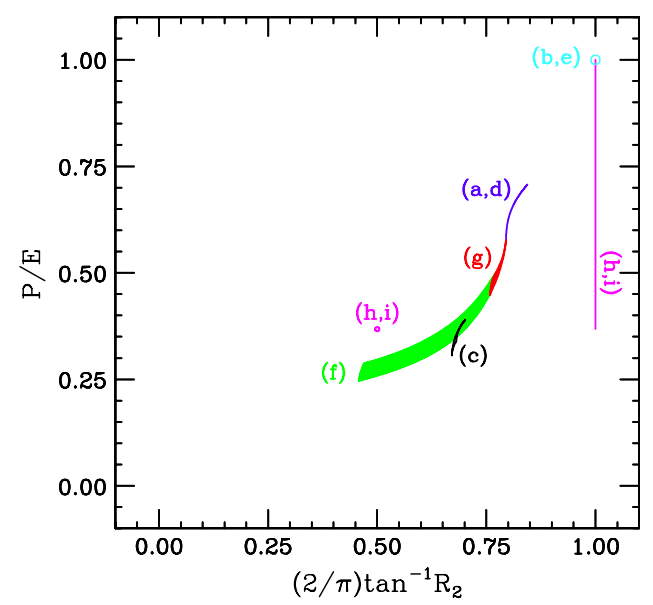

FIG. 4 (color online). The topology disambiguation diagram. The different color-coded regions delineate the range of values for $R_{2}$ and $P / E$ spanned by each decay topology from Fig. 2. 


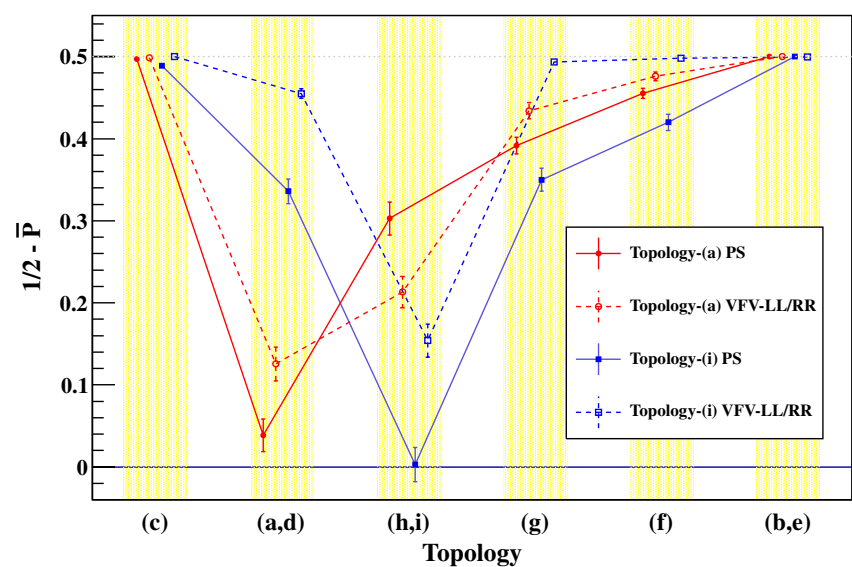

FIG. 5 (color online). Results from a quantitative topology disambiguation exercise using $\chi^{2}$ as test statistics.

distorted due to spin correlations [16]. In order to study the effect of spins, we repeat the two exercises for the case of purely left-handed $(L)$ or purely right-handed $(R)$ couplings of the fermions $B$ to the vector bosons $A$ and $C$. The results are displayed in Fig. 5 with open symbols. We see that, even though we were fitting to pure phase space formulas [17], the correct topologies are still singled out, as they provide the best fit to the data.

W. S. C. thanks the U.S. National Science Foundation, Grant No. NSF-PHY-0969510, the LHC Theory Initiative. D. K. acknowledges support from the LHC Theory Initiative Graduate Fellowship (NSF Grant No. PHY0969510). M.P. is supported by the CERN-Korea Fellowship through the National Research Foundation of Korea. Work supported in part by U.S. Department of Energy Grant No. DE-FG02-97ER41029.

[1] A. Barr and C. Lester, J. Phys. G 37, 123001 (2010).

[2] Y. Bai and H. -C. Cheng, J. High Energy Phys. 06 (2011) 021.

[3] E. Byckling and K. Kajantie, Particle Kinematics, (John Wiley \& Sons, New York, 1973).
[4] K. Agashe, D. Kim, D. G. E. Walker, and L. Zhu, Phys. Rev. D 84, 055020 (2011); G. F. Giudice, B. Gripaios, and R. Mahbubani, Phys. Rev. D 85, 075019 (2012).

[5] W. Cho, D. Kim, K. Matchev, and M. Park (to be published).

[6] We note that the resonance $A$ is, in general, allowed to be produced fully inclusively, with an arbitrary number of additional visible or invisible particles recoiling against $A$ in the event. This precludes us from using the $E_{T}$ measurement, since it will be corrupted by the invisible recoils, which leaves us with $m_{v_{1} v_{2}}$ as the only viable observable to study. The related combinatorial problem of partitioning the visible particles in the event was addressed in [2,7].

[7] M. Blanke, D. Curtin, and M. Perelstein, Phys. Rev. D 82, 035020 (2010); A. Rajaraman and F. Yu, Phys. Lett. B 700, 126 (2011); P. Baringer, K. Kong, M. McCaskey, and D. Noonan, J. High Energy Phys. 10 (2011) 101.

[8] Note that some of the event topologies in Fig. 2 involve effective higher-dimensional interactions $[9,10]$, which we assume to be pointlike, otherwise, effects of their mediators can be seen in other processes at the LHC.

[9] A. Birkedal, R. Group, and K. Matchev, arXiv:hep-ph/ 0507002.

[10] D. J. Phalen and A. Pierce, Phys. Rev. D 76, 075002 (2007); L. Edelhauser, W. Porod, and R. K. Singh, J. High Energy Phys. 08 (2010), 053; C. -Y. Chen and A. Freitas, J. High Energy Phys. 01 (2012) 124.

[11] I. Hinchliffe, F. E. Paige, M. D. Shapiro, J. Soderqvist, and W. Yao, Phys. Rev. D 55, 5520 (1997); K. Matchev, F. Moortgat, L. Pape, and M. Park, J. High Energy Phys. 08 (2009) 104.

[12] W. S. Cho, K. Choi, Y. G. Kim, and C. B. Park, Phys. Rev. Lett. 100, 171801 (2008).

[13] J. Alwall, A. Freitas, and O. Mattelaer, AIP Conf. Proc. 1200, 442 (2010).

[14] K. Agashe, D. Kim, M. Toharia, and D. G. E. Walker, Phys. Rev. D 82, 015007 (2010).

[15] T. Han, I.-W. Kim, and J. Song, Phys. Lett. B 693, 575 (2010).

[16] M. Burns, K. Kong, K. T. Matchev, and M. Park, J. High Energy Phys. 10 (2008) 081; L. Edelhauser, K. T. Matchev, and M. Park, J. High Energy Phys. 11 (2012) 006.

[17] Following the procedure in [16], one could derive analytical formulas for the invariant mass distributions in the presence of spin effects, but that is beyond the scope of the current Letter [5]. 